\title{
Tradução e adaptação cultural de questionário sobre liderança em Odontologia
}

\author{
Andressa Cartaxo de Almeida*; Fábio Luiz Cunha D'Assunção**; Talitha Rodrigues Ribeiro \\ Fernandes Pessoa ${ }^{* * * ;}$ Elsbeth Kalenderian****; Arturo Rodrigues Felinto*****; Helen Silva \\ Gonçalves******; Diana Santana Albuquerque ${ }^{* * * * * * *}$
}

\author{
* Mestre, Faculdade de Odontologia, da Universidade de \\ Pernambuco \\ ** Doutor, Professor Associado, Departamento de Odontologia \\ Restauradora da UFPB \\ *** Doutora, Professora Adjunta, Departamento de Clínica e \\ Odontologia Social da UFPB \\ **** Doutora, Presidente do Departamento de Odontologia Preventiva \\ e Social da Faculdade de Odontologia de São Francisco da \\ Universidade da Califórnia \\ ***** Mestre, Professor Assistente, Departamento de Administração da \\ UFPB \\ ****** Doutora, Professora Adjunta, Departamento de Administração da \\ UFPB
}

Recebido em 23/08/2017. Aprovado em 26/11/2017.

\begin{abstract}
RESUMO
O objetivo desse trabalho foi traduzir e adaptar para a cultura brasileira um questionário sobre habilidades de liderança de cirurgiões-dentistas. Para isso, o instrumento criado na Harvard School of Dental Medicine (HSDM) passou pelas etapas de tradução, retrotradução, adaptação transcultural a partir de uma reunião de experts no assunto e pré-teste com um grupo de cirurgiões-dentistas. Observou-se que alguns termos necessitaram ser adaptados para melhor interpretação, após o que o instrumento foi considerado de fácil compreensão por profissionais da área de interesse. $\mathrm{O}$ questionário necessita ainda de validação para que possa ser utilizado em pesquisas no Brasil.
\end{abstract}

Descritores: Liderança. Comparação Transcultural. Odontologia. Inquéritos e Questionários.

\section{INTRODUÇÃO}

Kalenderian et $a l .{ }^{1}$ descrevem liderança como a capacidade de determinar uma direção, alinhar, motivar e inspirar pessoas. O exercer de uma liderança eficaz é essencial para o avanço da profissão de cirurgião-dentista (CD), e o enfrentamento de novos desafios com base em realizações passadas leva à inovação e mudanças positivas $^{2,3}$.

Segundo as Diretrizes Curriculares Nacionais (DCN) para os cursos de Odontologia, durante a sua formação, o CD deve desenvolver competências e habilidades em liderança, estando apto para o trabalho em equipe 
multiprofissional, sempre tendo em vista o bemestar da comunidade 4 . A liderança envolve compromisso, responsabilidade, empatia, habilidade para tomada de decisões, se comunicar e gerenciar de forma efetiva e eficaz.

Taichman e Parkinson ${ }^{5}$ acreditam que o foco em liderança é um componente essencial que está faltando nos currículos de graduação em Odontologia, que a não formação e desenvolvimento de futuros líderes é um grave erro e coloca a profissão em risco. Alguns estudos já deixaram clara a falha na formação do $\mathrm{CD}$ em relação a aspectos de liderança ${ }^{3,5,6}$.

Até o momento, poucos relatos foram publicados a respeito das habilidades de liderança dos CD no Brasil. Além disso, é notória a escassez de um instrumento padronizado, atualizado e específico sobre o tema.

Para padronizar instrumentos de avaliação, principalmente questionários, desenvolvidos e utilizados em outro idioma, é necessário que seja realizada a sua adaptação transcultural. Esse processo envolve tradução, adaptação cultural e validação, transformando a aplicação do instrumento em um procedimento confiável, compreensível e adequado aos aspectos culturais da população em estudo ${ }^{7}$.

Como parte de um novo curso de liderança da Harvard School of Dental Medicine (HSDM), Kalenderian et $a{ }^{1}{ }^{1}$ desenvolveram um questionário sobre liderança para $\mathrm{CD}$. Esse instrumento mostrou ser uma ferramenta qualificada, que abrange várias competências relevantes de liderança e que leva o profissional a refletir e se autoavaliar. É um questionário versátil que pode ser aplicado em diversos momentos, como por exemplo antes ou após um treinamento dos profissionais em relação a essas habilidades, o que facilita a identificação de falhas nos processos de formação do profissional quanto aos aspectos abordados.

O objetivo desse estudo foi traduzir e adaptar esse questionário de liderança para o contexto brasileiro, a fim de obter um instrumento adequado para avaliar as habilidades e competências dos CD brasileiros em liderança e assim entender as necessidades e demandas sobre o tema.

\section{METODOLOGIA}

Para a validação transcultural do questionário foi seguida a metodologia preconizada por Guillemin, Bombardier e Beaton $^{8}$, que compreende as seguintes etapas: tradução, retrotradução (back-translation), avaliação de experts, pré-teste e validação propriamente dita (figura 1). A autorização para realizar o processo de validação transcultural do questionário foi obtida diretamente com a sua autora, Dr. Elisabeth Kalenderian, professora e presidente da University of California, em São Francisco, e professora convidada da HSDM.

\section{Apresentação do questionário}

O questionário é composto por uma lista com 15 habilidades de liderança, 8 perguntas, 2 frases indicando como responder às perguntas, uma escala do tipo Likert com 5 itens e uma escala de probabilidade com 3 itens, somando 33 componentes a serem traduzidos.

As perguntas do questionário são curtas e diretas. Em um primeiro momento é solicitado que o respondente avalie por meio de uma escala de 1 a 5, 15 habilidades de liderança, no qual 1 significa que o mesmo se avalia como péssimo e 5 excelente em relação à habilidade questionada; em seguida é perguntado quais das habilidades são mais e menos relevantes para ele; e qual a probabilidade de ele desenvolver essas habilidades de liderança; as últimas questões tratam sobre as percepções e anseios do respondente a respeito do que foi questionado (figura 2). 


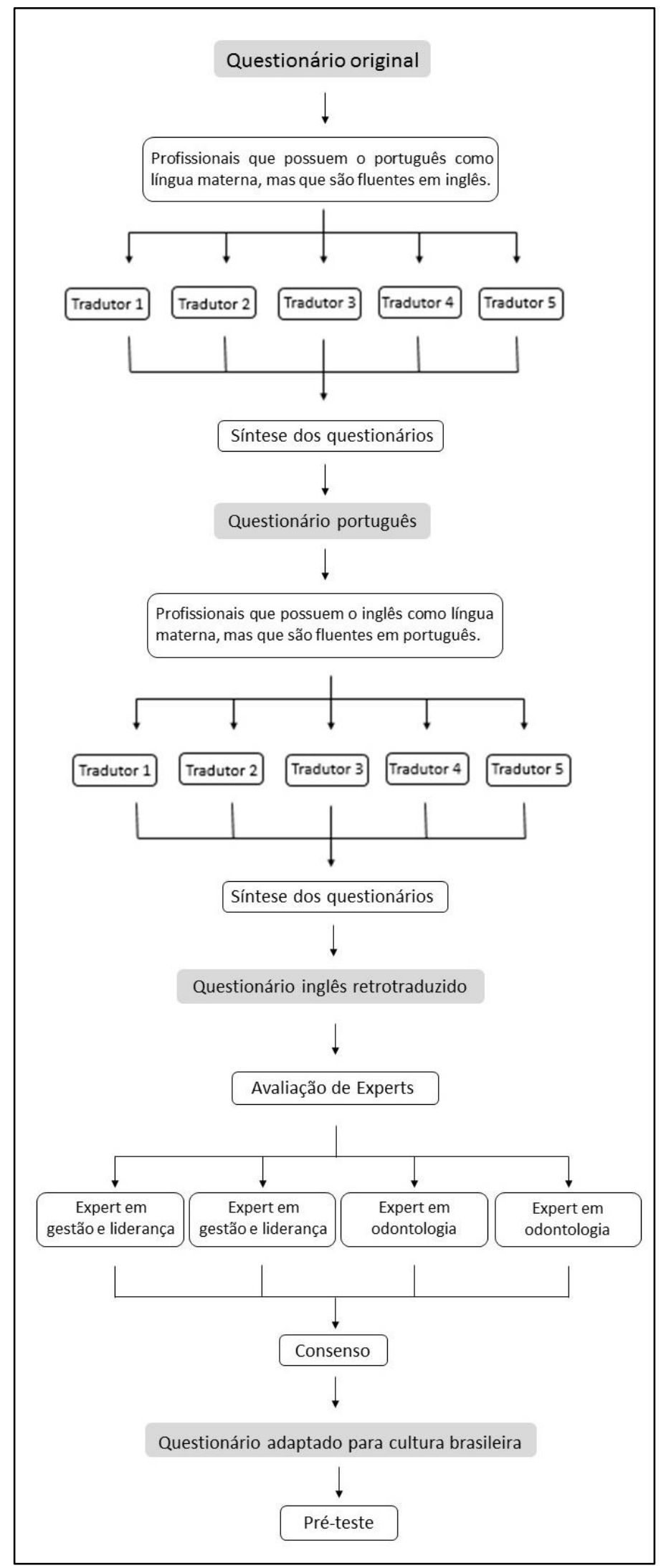

Figura 1. Diagrama das etapas de tradução, retrotradução, avaliação por experts e pré-teste

Revista da ABENO • 18(1):146-154, 2018 - DOI: https://doi.org/10.30979/rev.abeno.v18i1.503 


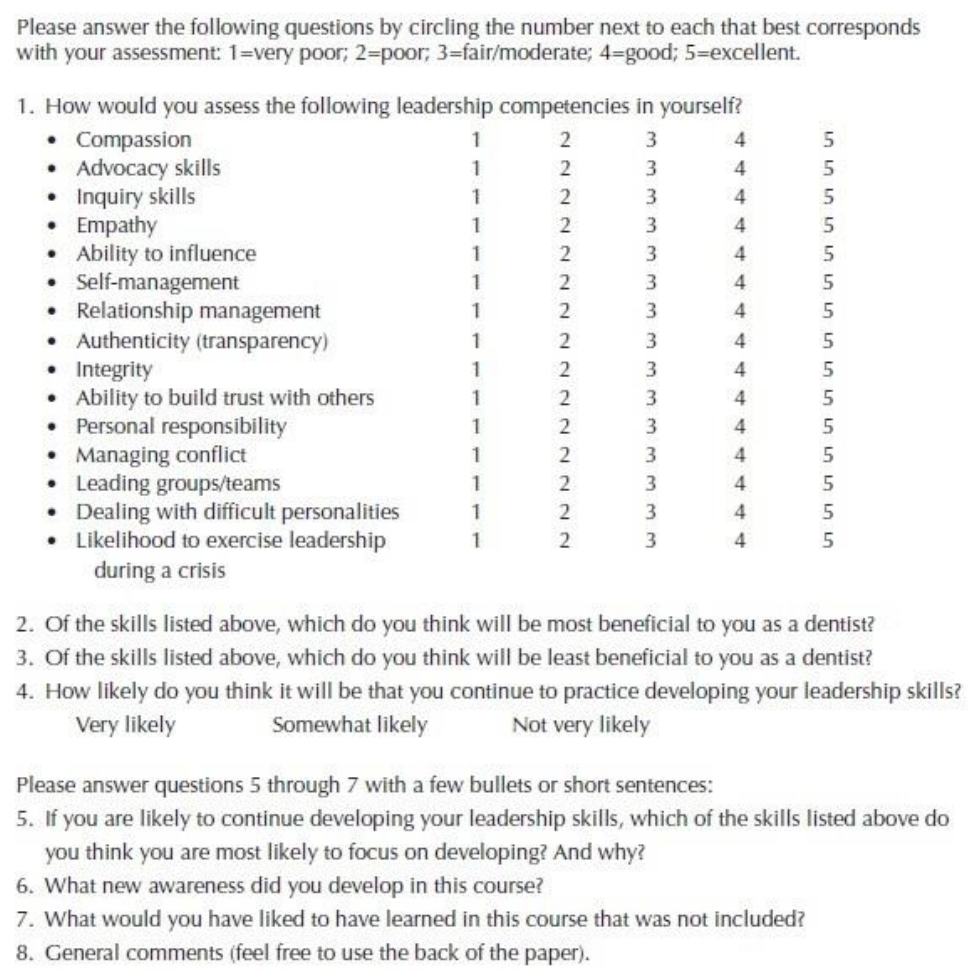

Figura 2. Instrumento original (Q0), em inglês.

\section{Tradução do instrumento}

\section{Tradução}

A finalidade dessa primeira etapa foi traduzir o questionário original (QO) de língua inglesa (US) para portuguesa (BR). Inicialmente, foram realizadas cinco traduções independentes. Essas traduções foram feitas para a língua portuguesa por pessoas maiores de idade, sem ser necessariamente da área de Odontologia, cuja língua materna fosse o português mas fluentes em inglês. Todos foram informados dos objetivos da tradução. Após a realização das traduções independentes, os pesquisadores realizaram uma análise conjunta do material, obtendo-se uma única tradução consensual do questionário do inglês, a partir dos termos que mais se repetiram, para o português (Q1).

\section{Retrotradução}

O procedimento adotado nessa etapa da tradução consistiu no envio do instrumento Q1 para cinco pessoas maiores de idade, não necessariamente da área de Odontologia, cuja língua materna fosse o inglês, com amplo domínio do português, com a finalidade que eles traduzissem o Q1 em português, para inglês novamente, sem que houvesse contato com o QO. Ressalta-se que esses profissionais foram informados dos objetivos da retrotradução. Com as cinco versões obtidas, os pesquisadores compararam o material e chegaram a um consenso, resultando em um novo questionário em inglês (Q2).

\section{Avaliação por Experts}

A análise das equivalências cultural, semântica, idiomática e conceitual foi realizada por um grupo de experts formado por quatro profissionais, sendo dois especializados na área de liderança e gestão, e dois $\mathrm{CD}$, todos com conhecimentos de inglês. Mediados pelos pesquisadores, todos discutiram e avaliaram cada 
um dos itens dos três questionários até chegarem a um consenso quanto a cada um dos itens componentes, obtendo um questionário traduzido para a língua portuguesa e adaptado para a cultura brasileira (QT).

\section{Pré-teste}

Com o QT em mãos, os pesquisadores fizeram um pré-teste com $30 \mathrm{CD}$, de acordo com o indicado por Malhotra ${ }^{9}$, apresentando o instrumento face a face, no qual os profissionais avaliaram a interpretação das instruções e das questões para avaliar se os objetivos do questionário seriam alcançados. Após essa etapa questionário final (QF) foi obtido (figura 3). A etapa de validação não foi realizada nesta fase.

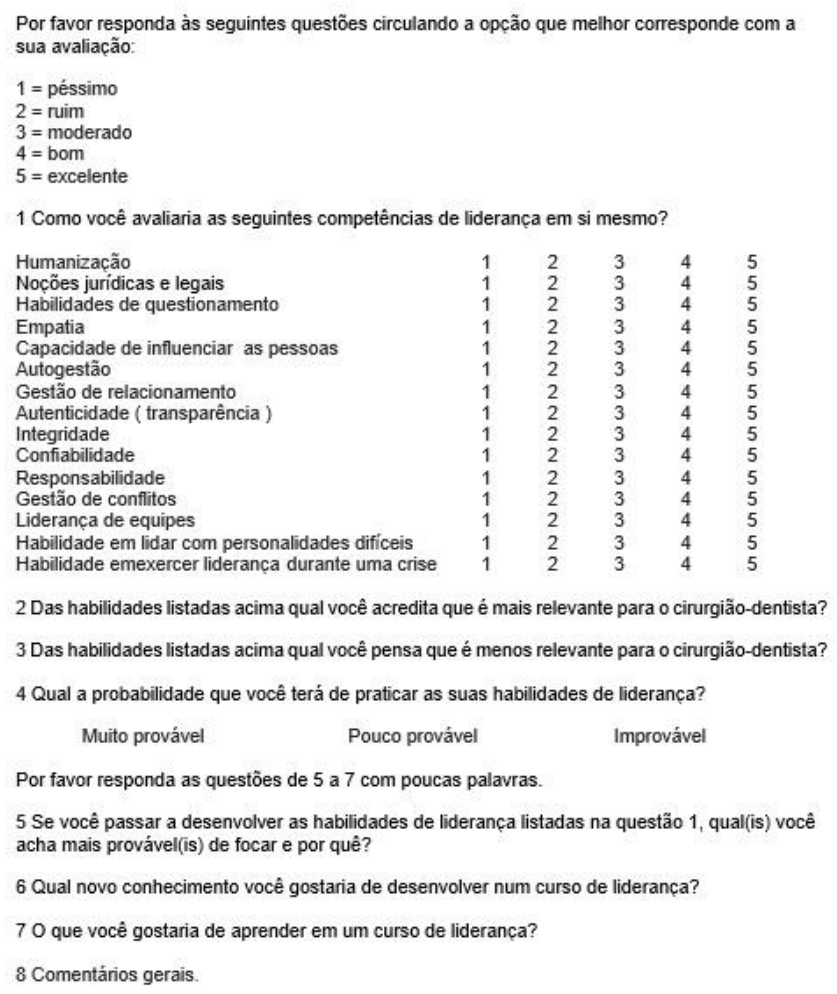

Figura 3. Instrumento traduzido para o português (Q1).

\section{RESULTADOS}

Após os processos de tradução e a reunião com os Experts para chegar a um consenso a respeito de um questionário traduzido e adaptado, foi possível observar equivalência semântico-idiomática, cultural e conceitual entre o instrumento original, as traduções e retrotraduções.

Alguns componentes do questionário não sofreram qualquer alteração, outros necessitaram de pequenas alterações de ordem gramatical, como inversão de palavras em frases ou substituição de alguns termos por sinônimos. Alterações maiores foram necessárias em poucos componentes, dois da lista das habilidades de liderança, sendo um a "Compaixão", alterada para "Humanização"; e o outro "Habilidades de advocacia" que foi alterado para "Noções jurídicas e legais”. Já os componentes “25, 30, 31 e 32 " eram perguntas voltadas para pessoas que haviam realizado o curso de liderança, como o público-alvo do QF não terá recebido essa 
qualificação especifica foi necessária a adaptação. Os graus das alterações realizadas estão dispostos na tabela 1.

Com o QT em mãos, foi realizado o pré- teste, que resultou em compreensão total das perguntas pelos 30 respondentes. Após essa etapa nenhum componente do questionário sofreu alteração, assim como mostra a tabela 2 .

Tabela 1. Alterações nos componentes do questionário.

\begin{tabular}{lcc}
\hline ALTERAÇÕES & $\mathbf{N}^{\mathbf{0}}$ DE COMPONENTES & $\mathbf{\%}$ \\
\hline Nenhuma & $14^{*}$ & 42,4 \\
Pequenas & $13^{* *}$ & 39,4 \\
Grandes & $6^{* * *}$ & 18,2 \\
\hline TOTAL & 33 & 100 \\
\hline$* 4,5,6,7,11,13,14,15,16,19,26,27,29$ e 33. & \\
$* * 1,2,3,10,12,17,18,20,21,22,23,24$ e 28. & \\
$* * * 8,9,25,30,31$ e 32. & &
\end{tabular}

Tabela 2. Praticabilidade* do QT após pré-teste.

\begin{tabular}{|c|c|c|c|c|c|c|}
\hline Questões & DT & DP & NTO & $\mathbf{C P}$ & CT & Total \\
\hline $\begin{array}{l}\text { Achei fácil entender as instruções } \\
\text { do questionário. }\end{array}$ & 0 & 0 & 0 & 0 & $\begin{array}{c}30 \\
(100 \%)\end{array}$ & $\begin{array}{c}30 \\
(100 \%)\end{array}$ \\
\hline $\begin{array}{l}\text { Achei fácil entender as questões } \\
\text { do questionário. }\end{array}$ & 0 & 0 & 0 & $\begin{array}{c}1 \\
(3,3 \%)\end{array}$ & $\begin{array}{c}29 \\
(96,7 \%)\end{array}$ & $\begin{array}{c}30 \\
(100 \%)\end{array}$ \\
\hline
\end{tabular}

DT - Discordo totalmente; DP - Discordo parcialmente; NTO - Não tenho opinião; CP - Concordo parcialmente; CT - Concordo totalmente. *Respostas ao Questionário de Avaliação de Praticabilidade ${ }^{10}$.

\section{DISCUSSÃO}

O processo de tradução, adaptação cultural e validação de questionários é complexo. Diversos instrumentos para pesquisa são utilizados no mundo, e é de fundamental importância padronizá-los, para que as discussões levem a resultados concretos ${ }^{11}$. Dessa forma é preciso que instrumentos já validados em outros idiomas passem por esse processo para serem utilizados em pesquisas no Brasil. Questionários padronizados levam à maior precisão nas avaliações coletivas, tanto em relação a questões acadêmicas, quanto epidemiológicas em saúde ${ }^{12}$.

Nesse estudo o processo ocorreu de forma sistematizada e seguiu as etapas preconizadas por metodologias já conhecidas ${ }^{8}$. O significado das palavras e os aspectos culturais do instrumento original e validado devem ser cuidadosamente considerados, pois a tradução literal pode resultar em um instrumento que não funciona da mesma forma para as diferentes populações ${ }^{13}$.

Na avaliação das equivalências semânticoidiomáticas, conceituais e culturais, realizada pelos experts foi possível identificar que a maioria das alterações foi simples. Algumas palavras e expressões foram alteradas apenas para facilitar o entendimento, assim como ocorre na maioria desses processos ${ }^{14-17}$.

$\mathrm{O}$ fato de o questionário original ter sido elaborado para ser aplicado após um curso específico de liderança fez com que algumas questões precisassem ser alteradas em relação ao tempo verbal. Foi possível adaptar de todas as questões. Caso algum pesquisador também queira aplicar o questionário no Brasil após realizar um curso de liderança, é possível readaptar o questionário para que ele fique adequado à situação.

Em relação aos termos das habilidades de 
liderança utilizados no questionário que sofreram grandes alterações, "Compaixão" foi um item que gerou bastante discussão na reunião dos experts. Observou-se que o mesmo poderia trazer a ideia de pena, de um paciente "coitadinho", não sendo assim um termo compatível com uma habilidade de liderança. No caso o termo que o substituiu foi "Humanização", bastante discutido nas políticas de saúde ${ }^{18,19}$.

A humanização também não deve ser interpretada apenas como agir com boa vontade para com o paciente, e pode ser melhor interpretada quando se define o que é desumano $^{19,20}$. Segundo Freire $^{21}$ o desumano ocorre quando há injustiça, exploração, opressão, violência e dominação das pessoas; o que não deve acontecer na relação profissional-paciente.

O componente "Habilidades em advocacia" também levantou alguns questionamentos, sendo substituído por "Noções jurídicas e legais". Entende-se que não é uma obrigação do $\mathrm{CD}$ advogar 22 , mas ele precisa saber seus direitos e deveres, assim como as aplicações legais da profissão, aspectos que estão presentes nas $\mathrm{DCN}^{4}$.

A fase de pré-teste é de fundamental importância para pesquisa. Segundo Malhotra ${ }^{9}$ é nessa fase em que é questionada a compreensão das perguntas, escalas para respostas, layout do questionário, entre outros, que possibilitam ao autor realizar ajustes e detectar incoerências que irão aumentar a validade do instrumento. Nesse estudo, após o pré-teste, foi possível observar que o instrumento foi bem compreendido, possui uma linguagem simples e, segundo os respondentes, está adequado para a cultura brasileira.

A investigação da condição das habilidades de liderança dos CD brasileiros é de fundamental importância para que estratégias sejam traçadas visando suprir possíveis falhas desses aspectos na formação do profissional. Morita et al. ${ }^{23}$ concluem que as competências de liderança, administração e gerenciamento são as que merecem maiores reparos na sua implantação na estrutura curricular dos cursos de Odontologia brasileiros.

Os estudantes de Odontologia atuais não são apenas os profissionais de amanhã, eles serão membros de conselhos municipais, diretores de saúde pública, educadores e líderes comunitários com uma incrível influência na saúde da população ${ }^{24-26}$. É através de programas de treinamento de liderança, desde a graduação, que eles devem desenvolver essas habilidades necessárias para o futuro exercício profissional ${ }^{2}$.

Outras profissões da Área da Saúde também discutem a percepção de liderança dos seus profissionais. Alguns estudos na área da Enfermagem mostraram que esses profissionais não foram bem preparados para desenvolver habilidades de liderança e que essa falha tornou clara a necessidade de incluir a liderança nos currículos de cursos graduação, assim como fortalecer programas de capacitação e educação permanente para aqueles que não foram preparados para serem líderes ${ }^{27-30}$. Na Medicina, uma sugestão é a da implantação de programas de desenvolvimento de liderança durante as residências, nas quais o médico está sendo moldado para ações que farão parte do seu cotidiano como profissional $^{31}$.

\section{CONCLUSÃO}

O questionário de liderança já revisado foi traduzido para o português e adaptado para a cultura brasileira de forma satisfatória, apresentando mínima necessidade de alterações, sendo bem compreendido pelos $\mathrm{CD}$. A próxima etapa será submeter o instrumento a um processo de validação psicométrica, de forma que possa ser utilizado em novas pesquisas.

\section{ABSTRACT \\ Translation and cultural adaptation of a}




\section{questionnaire about leadership in Dentistry}

The aim of this study was to translate and adapt a questionnaire on dentistry leadership skills to the Brazilian culture. For this purpose, the instrument created at the Harvard School of Dental Medicine (HSDM) went through the stages of translation, back-translation and crosscultural adaptation by an expert group to be submitted afterwards to a pre-test with a group of dentists. To facilitate a clear interpretation. It was observed that some terms needed to be adapted for better interpretation, after which the instrument was considered easy to understand by professionals of the area of interest. To use the questionnaire for research in Brazil, further psychometrc validation is required.

Descriptors: Leadership. Cross-Cultural Comparison. Dentistry. Surveys and Questionnaires.

\section{REFERÊNCIAS}

1. Kalenderian E, Skoulas A, Timothé P, Friedland B. Integrating leadership into a practice management curriculum for Dental students. J Dent Educ. 2010;74(5):464-71.

2. Victoroff KZ, Schneider K, Perry C. Leadership development for Dental students: what do students think? J Dent Educ. 2008;72(9):982-88.

3. Victoroff KZ, Schneider K, Perry C. Tomorrow's Leaders, Starting Today: A pilot leadership development program for Dental students. J Dent Educ. 2009;73(3):311-18.

4. Brasil. Resolução CNE/CES 3, de 19 de fevereiro de 2002. Institui Diretrizes Curriculares Nacionais do Curso de Graduação em Odontologia. Diário Oficial Da União, 04 de março de 2002.

5. Taichman RS, Parkinson JW, Nelson BA, Nordquist B, Ferguson-Young DC, Thompson JF. Leadership training for oral health professionals: a call to action. J Dent Educ. 2012;76(2):18591.

6. D'Assunção, FLC, De Almeida AC,
Kalenderian E. Knowledge of leadership and management: pilot study of student's perceptions of a dentistry course at a university in Brazil. Rev Abeno. 2015;15(2):28-37.

7. Sardinha A, Levitan MN, Lopes FL, Perna G, Esquivel G, Griez EJ, Nard AE. Tradução e adaptação transcultural do Questionário de Atividade Física Habitual. Rev Psiquiatr Clin. 2010;37(1):16-22.

8. Guillemin F, Bombardier C, Beaton D. Crosscultural adaptation of health-related quality of life measures: literature review and proposed guidelines. J Clin Epidemiol. 1993;46(12):1417-32.

9. Malhotra NK. Pesquisa de marketing: uma orientação aplicada. $4^{\text {a }}$ ed. Porto Alegre: Bookman; 2006.

10. Coluci MZO, Alexandre NMC. Development of a questionnaire to evaluate the usability of assessment instruments. Rev Enferm UERJ. 2009;17(3):378-82.

11. Bernardo EB, Catunda HL, de Oliveira MF, Lessa PR, Ribeiro SG, Pinheiro AK. Percurso metodológico para tradução e adaptação de escalas na área de saúde sexual e reprodutiva: uma revisão integrativa. Rev Bras Enferm. 2013 Jul-Aug;66(4):592-8.

12. Campolina AG, Ciconelli RM. Qualidade de vida e medidas de utilidade: parâmetros clínicos para as tomadas de decisão em saúde. Rev Panam Salud Publica. 2006;19(2):12836.

13. Beck CT, Bernal H, Froman RD. Methods to document semantic equivalence of a translated scale. Res Nurs Health. 2003;26(1):64-73.

14. Duarte PS, Miyazaki MCO, Ciconelli RM, Sesso R. Translation and cultural adaptation of the quality of life assessment instrument for chronic renal patients (KDQOL-SF TM). Rev Assoc Med Bras. 2003;49(4):375-81. 
15. Leal FDJ, Couto RC, Pitta GBB, Leite PTF, Costa LM, Higino WJ, Sousa MSCD. Tradução e adaptação cultural do Questionário Aberdeen para Veias Varicosas. J Vasc Bras. 2012;11(1):34-42.

16. De Souza AC, Milani D, Alexandre NMC. Adaptação cultural de um instrumento para avaliar a satisfação no trabalho. Rev Bras Saude Ocup. 2015;40(132):219-27.

17. Marchon SG \& Mendes Junior WV. Tradução e adaptação de um questionário elaborado para avaliar a segurança do paciente na atenção primária em saúde. Cad Saúde Pública. 2015;1(7):1395-402.

18. Benevides R., Passos E. A humanização como dimensão pública das políticas de saúde. Cienc Saúde Coletiva. 2005;10(3) :561-71.

19. Ferreira JA, Araújo GC. Humanização na Saúde: uma análise dos sentidos na óptica do trabalho cotidiano. Textos Contextos (Porto Alegre). 2014;13(1):199-213.

20. Gallian, DMC, Ponde LF, Ruiz R. Humanização, Humanismos e Humanidades: Problematizando conceitos e práticas no contexto da saúde no Brasil. Rev Int Hum Med. 2012;1(1):5-15.

21. Freire P. Pedagogia do oprimido. $42^{\mathrm{a}}$ ed. Rio de Janeiro: Paz e Terra; 2005.

22. da Silva RHA, de Oliveira MJ, Melani RFH, Oliveira RN. Responsabilidade civil do cirurgião-dentista: a importância do assistente técnico. R Dental Press Ortodon Ortop Facial. 2009;14(1):65-71.

23. Morita MC, Kriger L, de Carvalho ACP, Haddad AE. Implantação das Diretrizes Curriculares Nacionais em Odontologia; Maringá: Dental Press Editora; 2013.

24. Hammer DA, Nadershahi NA. Assessing the demand and preferred format of a student leadership development program at Pacific. J Dent Educ. 2011;75(8):1044-52.
25. Skoulas A, Kalenderian E. Leadership training for postdoctoral dental students. J Dent Educ. 2012;76(9):1156-66.

26. Kalenderian E, Taichman RS, Skoulas A, Nadershahi N, Victoroff KZ. Developing the next generation of leaders in oral health. $\mathrm{J}$ Dent Educ. 2013;77(11):1508-14.

27. de Assis Simões AL, Fávero N. Aprendizagem da liderança: opinião de enfermeiros sobre a formação acadêmica. Rev Latino-Am Enfermagem. 2000;8(3):91-6.

28. Strapasson MR, Medeiros CR. Liderança transformacional na enfermagem. Rev Bras Enferm. 2009;62(2):228-33.

29. Amestoy SC, Cestari ME, Thofehrn MB, Backes VMS, Milbrath VM, de Lima Trindade L. As percepções dos enfermeiros acerca da liderança. Rev Gaúcha Enferm. 2009;30(4):617-24.

30. Ávila VC, Amestoy SC, Porto AR, Thofehrn MB, Trindade LL, Figueira AB. Visão dos docentes de enfermagem sobre a formação de enfermeiros-líderes. Cogitare Enferm. 2012;17(4):621-7.

31. Blumenthal DM, Bernard K, Bohnen J, Bohmer R. Addressing the leadership gap in medicine: residents' need for systematic leadership development training. Acad Med. 2012;87(4):513-22.

Correspondência para

Andressa Cartaxo de Almeida

e-mail: andressacartaxodealmeida@gmail.com

Av. General Newton Cavalcanti, 1650 - Tabatinga

54756-220 Camaragibe/PE 